\title{
Wound-Healing Potential of Cucurbita moschata Duchesne Fruit Peel Extract in a Rat Model of Excision Wound Repair
}

\author{
Saba Shaygan $\left(\mathbb{D},{ }^{1}\right.$ Sajad Fakhri $\mathbb{D}^{2},{ }^{2}$ Gholamreza Bahrami $\mathbb{D}^{2},{ }^{2}$ Khodabakhsh Rashidi $\mathbb{D},{ }^{3}$ \\ and Mohammad Hosein Farzaei iD ${ }^{2}$ \\ ${ }^{1}$ Student Research Committee, Kermanshah University of Medical Sciences, Kermanshah, Iran \\ ${ }^{2}$ Pharmaceutical Sciences Research Center, Health Institute, Kermanshah University of Medical Sciences, Kermanshah, Iran \\ ${ }^{3}$ Research Center of Oils and Fats, Kermanshah University of Medical Sciences, Kermanshah, Iran \\ Correspondence should be addressed to Mohammad Hosein Farzaei; mh.farzaei@gmail.com
}

Saba Shaygan and Sajad Fakhri contributed equally to this work.

Received 21 December 2020; Revised 4 August 2021; Accepted 31 August 2021; Published 15 September 2021

Academic Editor: Abdeslam Jaafari

Copyright ( 2021 Saba Shaygan et al. This is an open access article distributed under the Creative Commons Attribution License, which permits unrestricted use, distribution, and reproduction in any medium, provided the original work is properly cited.

Background and Aim. Pumpkin (Cucurbita moschata Duchesne) is a medicinal plant with different pharmacological effects such as antioxidant, antidiabetic, hepatoprotective, and anticancer effects. In the present study, we aimed to investigate wound-healing activity of pumpkin fruit peel in a rat model of excision wound repair. Materials and Methods. Hydroalcoholic extractions of pumpkin fruit peel were obtained and used to prepare two different cold cream-based formulations, namely, $10 \%$ and $20 \%$ pumpkin peel extracts (PPEs). These formulations, phenytoin cream, and cold cream were topically used once daily for 14 days to compare their wound-healing effects in a rat model of excision wound repair. Wound sizes were monitored at different intervals. Skin tissue samples were subject to H\&E staining for histopathological analysis. Blood samples were also taken on day 14 to measure serum levels of nitrite. Results. Both 10\% and 20\% PPE formulations resulted in a significant reduction of wound sizes compared to positive and negative controls. Wound closure rate was estimated to be higher in 20\% PPE-treated rats. According to histopathological analysis, treatment with 20\% PPE improved parameters associated with efficient wound repair, including better regeneration of epidemic layer, higher density of dermis collagen fibers, and lower presence of inflammatory cells. Also, both formulations lowered serum concentrations of nitrite. Conclusion. Given the obtained data from our study, the hydroalcoholic extract of Cucurbita moschata Duchesne fruit peel is proposed to be effective in accelerating the process of excision wound repair partly due to its antioxidant effect in terms of decreasing nitrite concentration.

\section{Introduction}

As the largest organ in human, skin covers the external surface of the body, serving as a protective barrier against invasive pathogens and different types of damages. Skin is a multilayer organ that is directly subject to various harmful microbial, mechanical, thermal, and chemical influences causing skin wounds [1]. Since wound healing and ulcer management impose a substantial health burden, those are regarded as major socioeconomic problems in developing as well as developed countries [2]. Coming in an orderly sequence, wound healing involves a series of events, including homeostasis, inflammation, granulation, and finally scar formation. Upon skin injury, platelets convert fibrinogen to fibrin causing their aggregation and formation of a hemostatic plug which prevents further bleeding. Platelets also secrete several growth factors such as platelet-derived growth factor (PDGF) and transforming growth factor- $\beta$ (TGF- $\beta$ ) as well as adhesion molecules able to activate the cells in the surrounding area. During the inflammatory phase, neutrophils infiltrate the injury site and secrete multiple highly active antimicrobial mediators like reactive oxygen species (ROS), cationic peptides, and proteases which play important roles in wound cleaning and prevention of infections [3]. After two days, macrophages come on the scene and trigger or take part in number of processes, including digestion of cell debris and matrix elements [4], release of growth factors and cytokines, and activation of 
angiogenesis cascade, thereby leading to the entrance of inflammatory and endothelial cells [5]. As part of the granulation tissue formation phase, reepithelialization involves the migration of keratinocytes into the injured area. Besides, fibroblasts invade the wound site and synthesize new extracellular matrix (ECM). Granulation tissue contains proteoglycans, glycosaminoglycans, collagen III, fibronectin, vitronectin, and thrombospondin which promote neovascularization. In response to tremendous angiogenic responses, fibroblasts transform into myofibroblasts which bring together the margins of the wounds to decrease the wound size. Scar tissue formation is considered as the last phase of the wound healing characterized by decreased inflammation and capillary density, apoptosis of myofibroblasts, and replacement of the provisional matrix with a new collagenous matrix [6].

Augmenting evidence highlights the involvement of ROS as critical regulators of several phases of the woundhealing process. ROS are indeed implicated in all woundhealing processes as low levels of ROS are required to cope with invading microorganisms and also for cell survival signaling. However, excessive ROS production or impaired detoxification of ROS causes oxidative damage, which is the major cause of nonhealing chronic wounds. Therefore, a balance of ROS production and scavenging is needed for a timely and efficient wound healing [7].

Traditional-based remedies represent cost-effective, simple, and efficacious alternatives for different types of wounds (burn wounds, ulcers, and infected wounds) by exerting a wide range of therapeutic effects which evoke the process of healing [8]. Herbal-derived compounds are the most extensively used therapeutics for skin lesions. These therapies include the application of herbs, finished herbal products, and herbal preparations which contain biologically active compounds capable of stimulating the healing process. Nowadays, a wide variety of plants, from different parts of the world, are analyzed and applied to treat skin lesions [9-11]. Herbalbased products are prepared as extracts, emulsions, creams, and ointments. Pumpkin, with the scientific name Cucurbita moschata (C. moschata), belongs to the genus Cucurbita and the family Cucurbitaceae. Pumpkin is an economically important species with a high production rate. In recent years, it has received a lot of attention due to the health benefits of the compounds derivable from its seeds and fruits [12]. Studies on the chemical and pharmacological features of C. moschata extracts derived from its different parts (including seeds, fruits, and stems) have confirmed several biological activities including anticancer [13], antidiabetic [14], antiobesity [15], and hepatoprotective [16] effects. Pumpkin is a nutritionally rich fruit as it possesses $\gamma$-tocopherol and carotenoid [17]. Also, its peel contains various amino acids, including aspartic acid, glutamic acid, arginine, histidine, glycine, leucine, isoleucine, alanine, methionine, lysine, valine, serine, threonine, tyrosine, and phenylalanine. Wound-healing properties of C. moschata Duchesne fruit peel on a rat model of burn wound healing has been previously reported [18]. As ROS and oxidative stress play key roles in the process of wound healing, the critical modulatory activity of $C$. moschata lignan on ROS during the in vitro and in vivo studies [19] showed related promising results towards skin regeneration. Hence, C. moschata seem to be a hopeful candidate in improving wound closure by modulation of oxidative mediators.

The aim of the current study was to evaluate the woundhealing effects of the hydroalcoholic extract of C. moschata Duchesne fruit peel in a rat model of excision wound repair.

\section{Materials and Methods}

2.1. Chemicals. The chemicals used in this study, including ethanol and glycerin, were from Merck Company (Germany). Ketamine hydrochloride/xylazine hydrochloride solution, hematoxylin and eosin, naphthylethylenediamine dihydrochloride (NEDD), sulphanilamide, and phosphoric acid were from Sigma-Aldrich Chemical Company (USA). Phenytoin cream and cold cream were provided by Behvazan Company (IRAN). As defined, cold cream is a waterin-oil $(W / O)$ emulsification, usually produced from beeswax and several fragrances. All other chemicals were of highest analytical grade.

2.2. Plant Extraction. Pumpkin fruits were purchased from a fruit store located in Kermanshah city, Iran, in October 2019 and were further verified by herbarium experts of the Division of Botany, Department of Biology, Faculty of Science, Razi University, Kermanshah, Iran. The fruits were peeled and air-dried at room temperature. Then, using a mechanical grinder, air-dried peels were pulverized to a coarse powder. Extraction was carried at room temperature in a percolator. In brief, $150 \mathrm{gr}$ of coarse powder was transferred to a percolator and soaked in ethanol/ $\mathrm{H}_{2} \mathrm{O}(70: 30 \mathrm{v} / \mathrm{v})$ as the solvent. Our extraction procedure involved three consecutive $24 \mathrm{~h}$ extractions using a new solvent each time. The resultant extracts were then mixed together. The final extract was concentrated using a rotary evaporator at room temperature to obtain a viscous liquid. The resultant concentrated liquid was lyophilized to yield a dry powder extract designated as pumpkin peel extracts (PPEs).

2.3. Cream Preparation. Two types of formulations with different concentrations of PPE (10\% and $20 \%)$ were prepared in cold cream-based formulation as $10 \%$ and $20 \%$ PPEs, respectively. The cold cream-based formulations were stored in the refrigerator and were first kept at room temperature for half an hour before being used for wound treatment each time. Cold cream and phenytoin $1 \%$ cream were used as negative and positive controls, respectively.

2.4. Animals. Male Wistar rats $(n=24)$ weighing between 220 and $250 \mathrm{~g}$ were included in this study, divided into 4 groups $(n=6)$. The rats were individually (one rat per cage) housed under standard environmental conditions with $23 \pm 1^{\circ} \mathrm{C}$ temperature and light/dark cycles of $12 \mathrm{~h} / 12 \mathrm{~h}$ and fed with normal laboratory food and water ad libitum. The experimental study was conducted in accordance with the Guide for the Care and Use of Laboratory Animals and approved by the 
Committee of Animal Ethics of the Kermanshah University of Medical Sciences (IR.KUMS.REC.1398.400).

2.5. Creation of Excision Wound. The rats were anesthetized by intraperitoneal (i.p.) injection of ketamine/xylazine (80/ $10 \mathrm{mg} / \mathrm{kg}$ ). The back of the rats was cleaned and shaved. Using a $2.5 \mathrm{~cm} \times 2.5 \mathrm{~cm}$ coin, dorsal part was marked, and then, excision wound was created by removing a patch of skin.

2.6. Treatment Protocol. The animals were randomly divided into four groups (6 rats in each group): a treatment group receiving 10\% PPE cream; a treatment group receiving $20 \%$ PPE cream; a positive control group receiving phenytoin cream; and a negative control group receiving cold cream. The day of wound creation was assumed as zero, and the treatment procedure started $24 \mathrm{~h}$ after wound creation. Formulations were topically applied to cover the wound surface every $12 \mathrm{~h}$ for 14 days. The animals were sacrificed on day 14 . On day 14 before sacrifice, blood samples were taken from animals to assess serum levels of nitrite, as a circulating biomarker of oxidative stress.

2.7. Wound Contraction Rate. Wound contraction was screened by taking photographs using a digital camera at an equal distance from the wounds and right angle to their surfaces. The captured images were then analyzed by Image J software to measure the wound size (area). The wound closure rate was expressed as the percentage of decrease in the initial wound size (zero-day wound size). The wound closure percentage of each time point was calculated using the following formula: wound closure percentage $=(W A 0-W A t) / W A 0 \times 100$, where WAt shows the wound area at each time point (days 3, 7, 10, and 14) after treatment and WA0 means wound area on day 0.

2.8. Histopathological Evaluation. All rats were euthanized with i.p. administration of thiopental sodium [20]. Skin tissue samples from the wound areas were collected on day 14 and preserved in $10 \%$ formalin to evaluate the histological changes. Tissue sections underwent hematoxylin/eosin (H\&E) staining followed by microscopic imaging under $\times 100$ and $\times 400$ magnifications [21].

2.9. Nitrite Assay. In this assay, $100 \mu \mathrm{l}$ of serum samples were mixed with $50 \mu \mathrm{l}$ of sulfanilamide (dissolved in $\mathrm{H}_{3} \mathrm{PO}_{4} 5 \%$ ). After incubating at room temperature for $5 \mathrm{~min}, 50 \mu \mathrm{l}$ of NEDD solution $\left(0.1 \%\right.$ in $\left.\mathrm{H}_{2} \mathrm{O}\right)$ was added to each supernatant. Optical densities of the samples were measured at $540 \mathrm{~nm}$ with an ELISA reader within $30 \mathrm{~min}$. Nitrite concentration was measured using the nitrite standard curve.

2.10. Statistical Analysis. All data were expressed as mean values \pm standard deviation (S.D.). Data were analyzed by two-way repeated measure analysis of variance (ANOVA) followed by Tukey's post hoc test. A $p$ value of less than 0.05 was considered as a statistically significant difference.

\section{Results}

The yield of hydroalcoholic extraction was determined to be $63.12 \%$ of the total weight of the dried powder.

3.1. Wound Contraction Rate. To assess the effects of $10 \%$ and $20 \%$ extracts on healing of the excision wounds, we measured wound size at different time intervals. Our analysis showed $10 \%$ and $20 \%$ PPE could significantly decrease the wound size as compared to the negative control group starting from day 3 after surgery. Also, wound-healing activity of $20 \%$ PPE cream was significantly higher than 10\% PPE cream and phenytoin (positive control) at all the time intervals evaluated. The comparison of wound size between 10\% PPE-treated group and phenytoin-treated group revealed that on day 3, 10\% PPE did better while its effects on wound contraction rate on days 7 and 10 after surgery were estimated to be weaker than phenytoin. On the final day of evaluation (day 14), however, 10\% PPE showed comparable effect on wound closure rate with phenytoin. Figure 1 shows the macroscopic trends of wound healing, and Figure 2 has indicated associated statistical results.

3.2. Histopathological Analysis. For histological evaluation of wound area tissue samples on the last day, we used $\mathrm{H} \& \mathrm{E}$ staining. Figure 3 depicts different magnifications of tissue sections from treatment and control groups. Also, the skin tissue section of a normal skin is presented. In the negative control group, the epidermis was not regenerated in most parts. The dermis showed a detached and irregular structure with disappeared appendages as well as high infiltration of inflammatory cells. Also, scattered collagen bundles were observed as evidenced by a low staining. In 10\% PPE-treated group, a similar pattern of tissue damage was visible. However, in 20\% PPE- and phenytoin-treated groups (positive control), skin tissue damages were decreased to some extent. Wrinkled epidermis was extensively regenerated, and the dermis exhibited a high density of connective tissue collagen fibers with presence of normal cells.

3.3. Nitrite Assay. To investigate systemic antioxidant activity of PPE, we conducted the nitrite assay on serum samples. Our results were indicative of significantly lower serum concentrations of nitrite in the rats treated with both PPE extracts (10\% and 20\%) and phenytoin than in the negative control group, indicating a systemic antioxidant mechanism (Figure 4). The changes in the nitrite level are comparable with a normal nitrite level, about $25 \mu \mathrm{m}$ [22].

\section{Discussion}

The process of wound healing encompasses inflammation, reepithelialization, granulation, and angiogenesis that lead to wound contraction [23]. Wound healing in a shorter time 


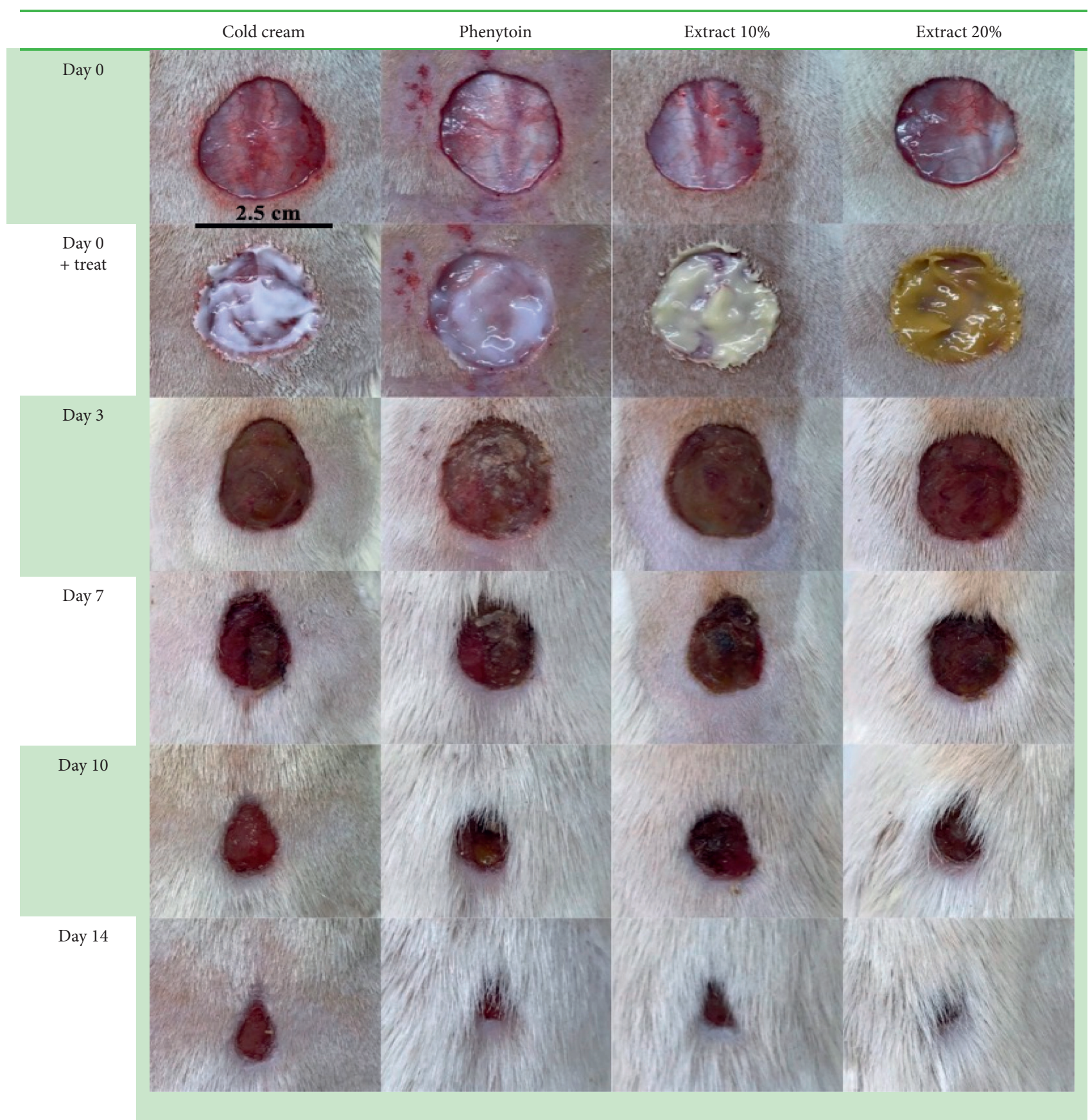

FIGURE 1: The macroscopic trends of wound healing in control, phenytoin-treated, extract $10 \%$, and extract $20 \%$.

and with less side effects is of the major aims of the current medicine. Various therapeutic strategies have been employed in this regard, including chemical and medicinal remedies and physical approaches such as laser therapy with the main goals of the whole treatments being effective, less toxic, and low-cost healing of the wound within a period as short as possible $[2,8,24]$. Despite the existence of a number of topical preparations in the market, there is still an apparent lack of an appropriate drug. Also, most of the medications or topical preparations available possess antimicrobial activities rather than wound repair effects and are associated with negative side effects and toxicity as is the case regarding silver sulfadiazine on fibroblasts [25]. In this respect, nearly one to three percent of novel medications are suggested to be effective on damaged or normal skin, while about one-third of herbal remedies are used for this purpose [23].

Medicinal plants could exert wound-healing effects due to their wide variety of different constituents and phytochemicals such as alkaloids, flavonoids, fatty acids, terpenoids, saponins, and phenolic compounds that are capable of improving the healing process. Phytochemicals can influence various stages of the wound-healing process through different mechanisms including upregulation of TGF- $\beta$, 


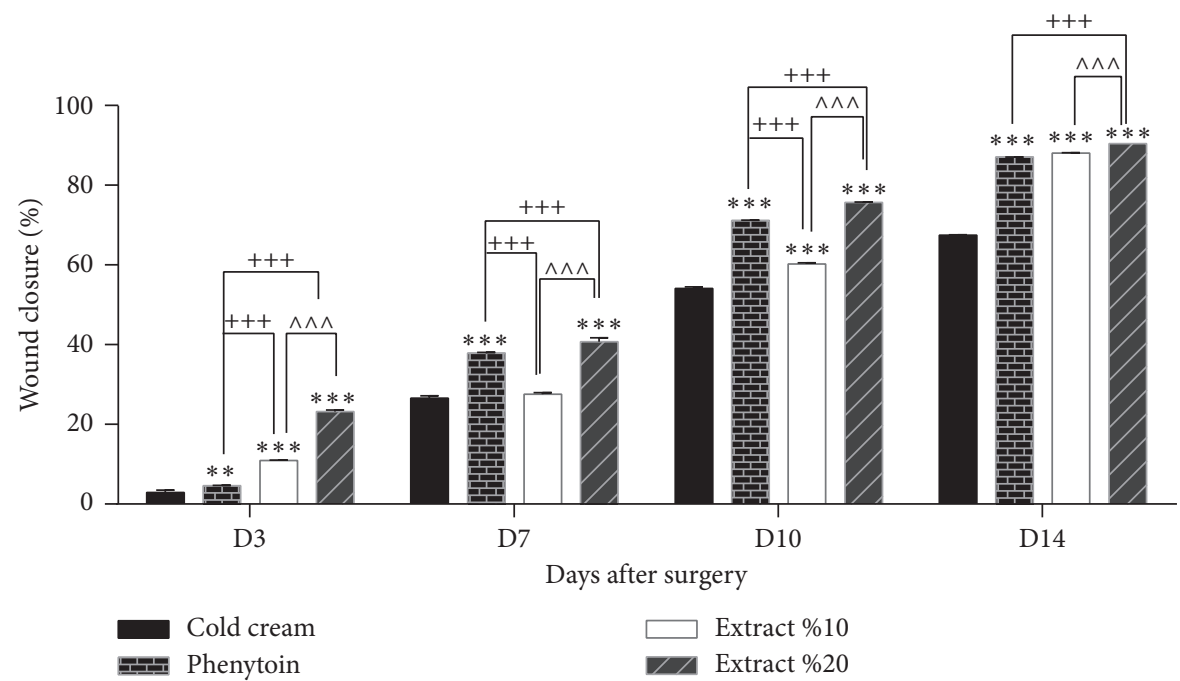

FiguRE 2: Wound closure percentage of different treated groups on different days after surgery. ${ }^{* *} P<0.05$ and ${ }^{* * *} P<0.001$, vs. cold cream; ${ }^{+++} p<0.001$, vs. phenytoin; and ${ }^{\wedge \wedge} P<0.001$, vs. extract $10 \%$.

vascular endothelial growth factor (VEGF), monocyte chemoattractant protein-1 (MCP-1), and interleukin- (IL-) 1 , as well as downregulation of NO and ROS. They also act through enhancing antioxidant power of tissue in the inflammatory phase, increasing matrix metalloproteinase (MMP) and proliferation of endothelial cells during the reepithelialization phase, improving the proliferation of damaged tissue cells in the granulation phase, and boosting angiogenesis by increasing TGF- $\beta$ and VEGF that finally lead to a reduction in wound contraction time [23].

In this study, we showed wound-healing activity of the hydroalcoholic extract of C. moschata Duchesne fruit peel in a rat model of excision wound repair. Also, we demonstrated the antioxidant potential of the pumpkin hydroalcoholic extract by nitrite assay. Based on wound closure results, $10 \%$ and 20\% PPE cream could accelerate the process of wound healing as compared to the negative control. Also, 20\% PPE cream exhibited more potent effects than 10\% PPE. In a mechanistic point of view, the wound-healing activity of C. moschata peel extract could be attributed to its high mucilage content and presence of different constituents such as flavonoids and phenolic compounds that are able to accelerate wound healing as well as its antioxidant power [18]. The most relevant study to our study is that of Bahramsoltani et al. investigating burn wound-healing activity of the hydroalcoholic extract of C. moschata Duchesne fruit peel. They elucidated beneficial effects of pumpkin peel extract (PPE) on a rat model of burn wound healing [18]. As shown by their study, PPE has a high mucilage content. Accumulating evidence suggests that a moist environment supports wound healing through several mechanisms, including stimulation of reepithelialization, angiogenesis, keratinocyte migration, and induction of hypoxia inducible factor-1 (HIF-1) that results in the production of endogenous stimulants of wound healing [26]. Thus, high mucilage content can partly account for the ameliorating effects of our PPE on excision wounds. Another group of phytochemicals accounting for the beneficial effects of our PPE includes flavonoids and phenolic compounds as the ethanolic extract of C. moschata Duchesne has been shown to contain these compounds [27]. There are several studies on the woundhealing activities of different parts of pumpkin including the study by Ben Halima and colleagues conducted on pumpkin (Cucurbita peop L.) seed oil [28]. Dried seed of pumpkin contains oil from $40 \%$ to $50 \%$ which is also shown to be rich in polyunsaturated fatty acids [29]. In their in vivo study, pumpkin seed oil did significantly better in healing the skin wounds than the reference group receiving Cicaflora cream (an emulsion containing 10\% of Mimosa tenuiflora extract). The group treated with pumpkin seed oil experienced full reepithelialization [28].

From the mechanistic point of view, regulation of ROS levels in wounded skin is necessary for an efficient wound repair. ROS, on the one hand, can contribute to the clearance of invading bacteria and are also key regulators of numerous intracellular signaling pathways at physiological concentrations. On the other hand, augmented levels of ROS can lead to cell damage and delay the process of wound repair [30]. In this regard, NO (as a type of ROS) also plays a dual role. Its high concentration can cause oxidative damage and hinder wound repair while it is also involved in cell proliferation, collagen formation, and wound contraction [31]. Our study showed that PPE can decrease serum nitrite levels, reflecting its systemic antioxidant activity to precipitate wound repair. In line with our results, Bahramsoltani et al. reported the antioxidant potential of PPE in terms of decreasing biomarkers of tissue oxidative stress. Based on their results, treatment with $10 \%$ and $20 \%$ PPE significantly lowered lipid peroxidation in skin tissues. Also, 20\% PPE could augment total antioxidant power (TAP) of the damaged tissues. Additionally, in vitro evaluations including 1,1-diphenyl-2-picrylhydrazyl (DPPH) radical scavenging and ferric-reducing antioxidant power (FRAP) tests were also indicative of antioxidant properties of the extract [18]. In another study, the pumpkin extract was shown to upregulate the activities of antioxidant enzymes such as 

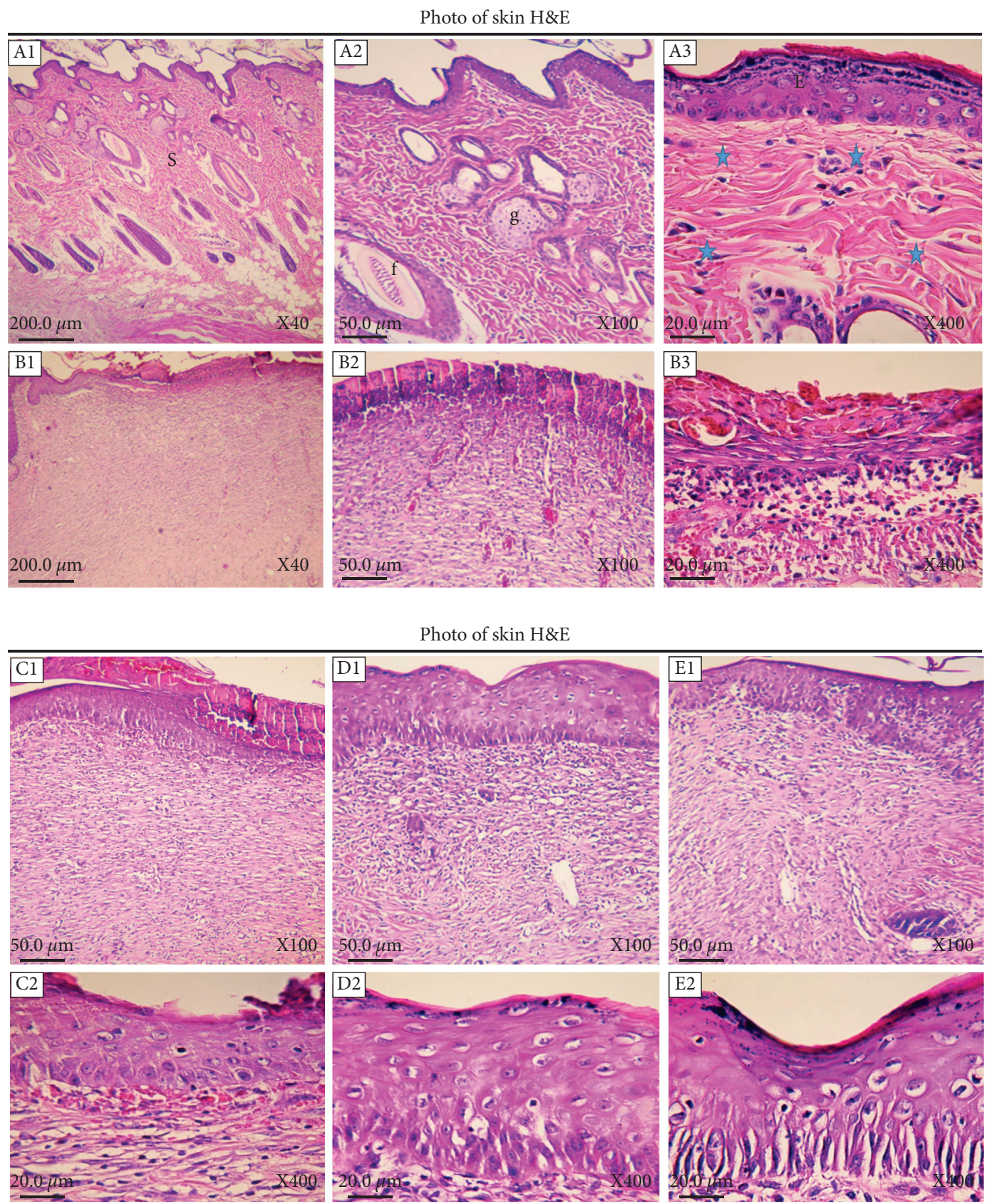

Figure 3: Histopathological evaluation of skin. (A1-A3) Normal healthy skin is a thick tissue (S) anatomically comprising two main segments of the epidermis and dermis. The epidermis consists of a keratinized stratified squamous epithelium (E), while the dermis is a connective tissue with different appendages including hair follicles (f), fat glands (g), and a variety of cells and regular connective tissue arrangements (asterisk). (B1-B3) $\times 40, \times 100$, and $\times 400$ magnifications of skin tissue sections of the negative control group, respectively. (C1, C2) $\times 100$ and $\times 400$ images of skin tissue from the $10 \%$ PPE-treated group. (D1, D2) $\times 100$ and $\times 400$ magnifications of skin tissue from the $20 \%$ PPE-treated group. (E1, E2) $\times 100$ and $\times 400$ images of the phenytoin-treated group.

glutathione peroxidase (GSH-Px) and superoxide dismutase (SOD) and also repressed lipid peroxidation in mice [32]. In accordance with these findings, it has been demonstrated that systemic administration of the pumpkin extract reduces malondialdehyde (MDA) and upregulates liver GSH-Px and SOD [33]. Also, investigations have confirmed antioxidant properties of pumpkin seed oil and fruit extract [34].

Nowadays, the fabrication and administration of novel delivery systems have also paved the road in wound healing.
In this line, using the encapsulation of epidermal growth factor in gelatin-alginate coacervates could significantly reduce the wound area by suppressing inflammatory cytokines (e.g., IL-1, IL-6, and TNF- $\alpha$ ) [35].

Taken together, our study showed wound-healing activity of C. moschata Duchesne fruit peel extract in a rat model of excision wound repair. Also, as a mechanism of action, we showed the antioxidant capability of the hydroalcoholic extract of C. moschata Duchesne as reflected 


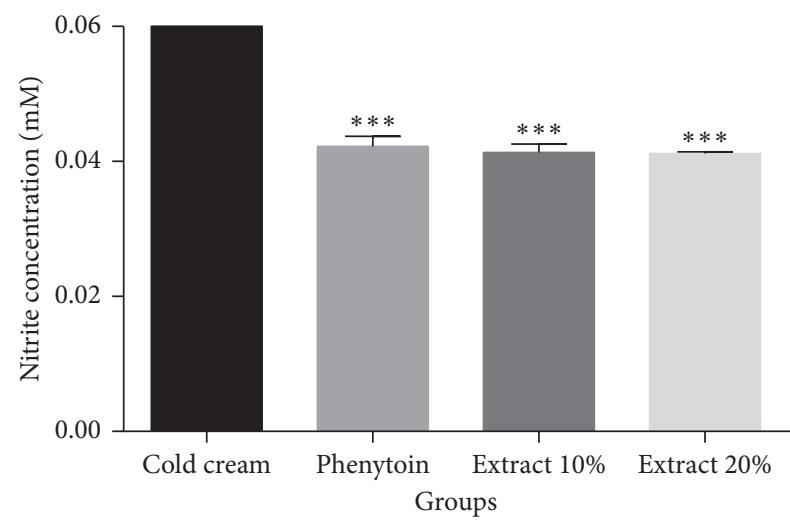

FIGURE 4: Nitrite concentrations (mm) of serum samples taken on day 14 after wound creation in each group. Cold cream: negative control group; phenytoin: phenytoin-treated group; extract $10 \%$ : 10\% PPE-treated group; extract 20\%: 20\% PPE-treated group. ${ }^{* * *} P<0.001$, vs. cold cream.

by reduction in nitrite levels in serum samples of the treated rats. Our data shed more light on the wound-healing activity of the peel extract of pumpkin. However, further supportive experimental and clinical investigations are required to approve the peel extract of this kitchen fruit as a woundhealing remedy.

\section{Data Availability}

The datasets used in the present study are available from the corresponding authors on reasonable request.

\section{Conflicts of Interest}

The authors declare no conflicts of interest.

\section{Authors' Contributions}

Saba Shaygan and Sajad Fakhri contributed equally to this work.

\section{Acknowledgments}

This research was supported by the Kermanshah University of Medical Sciences (Grant no. 980565), Kermanshah, Iran.

\section{References}

[1] S. MacNeil, "Progress and opportunities for tissue-engineered skin," Nature, vol. 445, no. 7130, pp. 874-880, 2007.

[2] A. V. Singh, A. S. Aditi, W. N. Gade, T. Vats, C. Lenardi, and P. Milani, "Nanomaterials: new generation therapeutics in wound healing and tissue repair," Current Nanoscience, vol. 6, no. 6 , pp. $577-586,2010$.

[3] C. Caravaggi, R. De Giglio, C. Pritelli et al., "HYAFF 11-based autologous dermal and epidermal grafts in the treatment of noninfected diabetic plantar and dorsal foot ulcers: a prospective, multicenter, controlled, randomized clinical trial," Diabetes Care, vol. 26, no. 10, pp. 2853-2859, 2003.

[4] S. J. Leibovich and R. Ross, "The role of the macrophage in wound repair: a study with hydrocortisone and antimacrophage serum," American Journal of Pathology, vol. 78, no. 1, pp. 71-100, 1975.

[5] P. J. Polverini, R. S. Cotran, M. A. Gimbrone, and E. R. Unanue, "Activated macrophages induce vascular proliferation," Nature, vol. 269, no. 5631, pp. 804-806, 1977.

[6] F. Groeber, M. Holeiter, M. Hampel, S. Hinderer, and K. Schenke-Layland, "Skin tissue engineering-in vivo and in vitro applications," Advanced Drug Delivery Reviews, vol. 63, no. 4-5, pp. 352-366, 2011.

[7] M. Cano Sanchez, S. Lancel, E. Boulanger, and R. Neviere, "Targeting oxidative stress and mitochondrial dysfunction in the treatment of impaired wound healing: a systematic review," Antioxidants, vol. 7, no. 8, p. 98, 2018.

[8] R. F. Pereira and P. J. Bártolo, "Traditional therapies for skin wound healing," Advances in Wound Care, vol. 5, no. 5, pp. 208-229, 2016.

[9] S. Vujanovic and J. Vujanovic, "Bioresources in the pharmacotherapy and healing of burns: a mini-review," Burns, vol. 39, no. 6, pp. 1031-1038, 2013.

[10] Y. Sharma, G. Jeyabalan, and R. Singh, "Potential wound healing agents from medicinal plants: a review," Pharmacologia, vol. 4, no. 5, pp. 349-358, 2013.

[11] M. Ayyanar and S. Ignacimuthu, "Herbal medicines for wound healing among tribal people in southern India: ethnobotanical and scientific evidences," International Journal of Applied Research in Natural Products, vol. 2, no. 3, pp. 29-42, 2009.

[12] S.-Y. Wang, W.-C. Huang, C.-C. Liu et al., "Pumpkin (Cucurbita moschata) fruit extract improves physical fatigue and exercise performance in mice," Molecules, vol. 17, no. 10, pp. 11864-11876, 2012.

[13] B. Zhang, H. Huang, J. Xie et al., "Cucurmosin induces apoptosis of BxPC-3 human pancreatic cancer cells via inactivation of the EGFR signaling pathway," Oncology Reports, vol. 27, no. 3, pp. 891-897, 2012.

[14] Z. Jiang and Q. Du, "Glucose-lowering activity of novel tetrasaccharide glyceroglycolipids from the fruits of Cucurbita moschata," Bioorganic \& Medicinal Chemistry Letters, vol. 21, no. 3, pp. 1001-1003, 2011

[15] J. Lee, D. Kim, J. Choi et al., "Dehydrodiconiferyl alcohol isolated from Cucurbita moschata shows anti-adipogenic and anti-lipogenic effects in 3T3-L1 cells and primary mouse embryonic fibroblasts," Journal of Biological Chemistry, vol. 287, no. 12, pp. 8839-8851, 2012.

[16] M. Makni, H. Fetoui, N. K. Gargouri et al., "Hypolipidemic and hepatoprotective effects of flax and pumpkin seed mixture rich in $\omega-3$ and $\omega-6$ fatty acids in hypercholesterolemic rats," Food and Chemical Toxicology, vol. 46, no. 12, pp. 3714-3720, 2008.

[17] C. H. Azevedo-Meleiro and D. B. Rodriguez-Amaya, "Qualitative and quantitative differences in carotenoid composition among Cucurbita moschata, Cucurbita maxima, and Cucurbita pepo," Journal of Agricultural and Food Chemistry, vol. 55, no. 10, pp. 4027-4033, 2007.

[18] R. Bahramsoltani, M. H. Farzaei, A. H. Abdolghaffari et al., "Evaluation of phytochemicals, antioxidant and burn wound healing activities of Cucurbita moschata duchesne fruit peel," Iranian Journal of Basic Medical Sciences, vol. 20, no. 7, pp. 798-805, 2017.

[19] J. Lee, J. Choi, and S. Kim, "Effective suppression of proinflammatory molecules by DHCA via IKK-NF- $\kappa$ B pathway, in vitro and in vivo," British Journal of Pharmacology, vol. 172, no. 13, pp. 3353-3369, 2015.

[20] K. K. Zatroch, C. G. Knight, J. N. Reimer, and D. S. Pang, "Refinement of intraperitoneal injection of sodium 
pentobarbital for euthanasia in laboratory rats (Rattus norvegicus)," BMC Veterinary Research, vol. 13, no. 1, pp. 1-7, 2016.

[21] J. D. Bancroft and M. Gamble, Theory and Practice of Histological Techniques, Elsevier Health Sciences, Amsterdam, Netherlands, 2008.

[22] A. Villegas-Bastida, R. Torres-Rosas, L. A. Arriaga-Pizano, J. Flores-Estrada, A. Gustavo-Acosta, and M. A. MorenoEutimio, "Electrical stimulation at the ST36 acupoint protects against sepsis lethality and reduces serum TNF levels through vagus nerve- and catecholamine-dependent mechanisms," Evidence-Based Complementary and Alternative Medicine, vol. 2014, Article ID 451674, 8 pages, 2014.

[23] R. Bahramsoltani, M. H. Farzaei, and R. Rahimi, "Medicinal plants and their natural components as future drugs for the treatment of burn wounds: an integrative review," Archives of Dermatological Research, vol. 306, no. 7, pp. 601-617, 2014.

[24] J. M. Reinke and H. Sorg, "Wound repair and regeneration," European Surgical Research, vol. 49, no. 1, pp. 35-43, 2012.

[25] G. Hassanzadeh, F. Hajmanouchehri, A. Roi et al., "Comparing effects of silver sulfadiazine, sucralfate and Brassica oleracea extract on burn wound healing," Life Science Journal, vol. 10, pp. 104-113, 2013.

[26] K. C. Broussard and J. G. Powers, "Wound dressings: selecting the most appropriate type," American Journal of Clinical Dermatology, vol. 14, no. 6, pp. 449-459, 2013.

[27] U. Chonoko and A. Rufai, "Phytochemical screening and antibacterial activity of Cucurbita pepo (pumpkin) against Staphylococcus aureus and Salmonella typhi," Bayero Journal of Pure and Applied Sciences, vol. 4, no. 1, pp. 145-147, 2011.

[28] S. Bardaa, N. Ben Halima, F. Aloui et al., "Oil from pumpkin (Cucurbita pepo L.) seeds: evaluation of its functional properties on wound healing in rats," Lipids in Health and Disease, vol. 15, no. 1, pp. 73-12, 2016.

[29] N. Poljšak, S. Kreft, and N. Kočevar Glavač, "Vegetable butters and oils in skin wound healing: scientific evidence for new opportunities in dermatology," Phytotherapy Research, vol. 34, no. 2, pp. 254-269, 2020.

[30] U. Auf Dem Keller, A. Kümin, S. Braun, and S. Werner, "Reactive oxygen species and their detoxification in healing skin wounds," Journal of Investigative Dermatology Symposium Proceedings, vol. 11, no. 1, pp. 106-111, 2006.

[31] M. B. Witte and A. Barbul, "Role of nitric oxide in wound repair," The American Journal of Surgery, vol. 183, no. 4, pp. 406-412, 2002.

[32] C. Dang, "Effect of pumpkin distillable subject on lipid peroxidation and the activity of antioxidative enzyme induced by plumbum in mouse," Chinese Journal of Clinical Rehabilitation, vol. 8, pp. 4378-4379, 2004.

[33] H. C. Xia, F. Li, Z. Li, and Z. C. Zhang, "Purification and characterization of moschatin, a novel type I ribosomeinactivating protein from the mature seeds of pumpkin (Cucurbita moschata), and preparation of its immunotoxin against human melanoma cells," Cell Research, vol. 13, no. 5, pp. 369-374, 2003.

[34] A. Azizah, K. Wee, O. Azizah, and M. Azizah, "Effect of boiling and stir frying on total phenolics, carotenoids and radical scavenging activity of pumpkin (Cucurbita moschato)," International Food Research Journal, vol. 16, no. 1, pp. 45-51, 2009.

[35] S. Jeong, B. Kim, M. Park, E. Ban, S.-H. Lee, and A. Kim, "Improved diabetic wound healing by EGF encapsulation in gelatin-alginate coacervates," Pharmaceutics, vol. 12, no. 4, p. 334, 2020. 